\title{
Una evaluación retrospectiva de la interdependencia económica entre México y Estados Unidos
}

\author{
María del Rosío Barajas-Escamilla * \\ Marcela Martínez \\ MARITZA SOTOMAYOR ${ }^{* * *}$
}

\section{RESUMEN}

En este artículo se analiza la evolución de la interdependencia económica México-Estados Unidos, aplicando índices de concentración de exportaciones e importaciones para el periodo 1980-2010. Los índices evidencian un alto grado de interdependencia asimétrica, que la integración comercial no ha resuelto, aun cuando México se ha esforzado por diversificar su comercio.

Palabras clave: sector externo, inversión extranjera directa, TLCAN, liberalización comercial, integración económica.

\section{ABSTRACT}

This article analyzes the evolution of Mexico-U.S. economic interdependence by applying export and import concentration indices for the years between 1980 and 2010. The indices show a high degree of asymmetrical interdependence, which has not been resolved by trade integration, even though Mexico has made efforts to diversify its trade.

Key words: external sector, foreign direct investment, NAFTA, trade liberalization, economic integration

\footnotetext{
* Departamento de Estudios Sociales, Colegio de la Frontera Norte, rbarajas@colef.mx.

** Departamento de Proyectos Especiales, m3vmm@hotmail.com.

*** Department of Finance and Economics, Utah Valley University, maritza.sotomayor@uvu.edu.
} 


\section{INTRODUCCIÓN}

El objetivo de este trabajo es analizar los efectos del comercio bilateral sobre la interdependencia económica entre México y Estados Unidos para el periodo 1980-2010, la cual se pensaba se profundizaría debido a la integración comercial iniciada con la firma del Tratado de Libre Comercio de América del Norte (TLCAN).

Este artículo presenta una evaluación retrospectiva de los flujos de comercio bilateral previos y posteriores al tratado. Para ello, se discuten los trabajos teórico-empíricos publicados sobre el tema y se utiliza el marco teórico-metodológico de Ming Zhang (1995), quien sostiene que la interdependencia económica tiene la capacidad de disminuir el conflicto entre países. Al ser la de México y Estados Unidos una relación de comercio norte-sur, se hubiera esperado una mayor probabilidad de que se establecieran relaciones de conflicto, las cuales un acuerdo de libre comercio podría resolver o por lo menos aminorar (Barbieri, 1996). La contribución de este documento se da a partir de la discusión de los textos teóricos y de las propuestas de mediciones cuantitativas de la interdependencia, que van más allá del análisis de los flujos de comercio. En este sentido, se propone complementar el análisis con la evaluación de la inversión extranjera directa (IED) -que se entiende como parte de los flujos de capital entre países- y del comercio entre fronteras, variables insoslayables en la dinámica que se establece entre ambos países.

Este artículo se organiza de la siguiente forma: en un primer apartado, se hace una revisión teórica del concepto de interdependencia y su evolución, la cual se respalda por la discusión de una serie de autores que han desarrollado esfuerzos de medición de la interdependencia. En el segundo apartado, se explica el modelo teórico-metodológico del cual se parte, explicando la similitud y diferencia del planteamiento de Zhang (1995). En el tercer apartado, se realiza el análisis de resultados, considerando las siguientes variables: importaciones, exportaciones, inversión extranjera directa y comercio entre fronteras. Finalmente, se presentan algunas reflexiones y comentarios finales.

\section{REVISIÓN DE LA LITERATURA TEÓRICO-EMPÍRICA}

El concepto de interdependencia económica fue originalmente acuñado por Hirshman (1945), para explicar las relaciones de dependencia que se establecen entre los países, a partir de su comercio internacional. Dicho concepto se recuperó durante los años setenta y tuvo relevancia de nuevo a principios de los noventa gracias a autores como Keohane y Nye (1989), quienes recobraron el concepto y lo utilizan para proponer que, frente a la nueva configuración geopolítica del mundo y ante el creciente proceso 
de globalización, las hegemonías no se definirían más a partir de las guerras, sino del comercio, y ello los llevó a profundizar más en el desarrollo de la teoría de interdependencia, incorporando conceptos como cooperación, conflicto, actores, sensibilidad y vulnerabilidad.

Asimismo, los autores antes mencionados analizaron la relación entre interdependencia-cooperación y conflicto, señalando que si bien la relación de interdependencia económica no resolvía de tajo el conflicto, sí estimulaba la cooperación y con ello reducía el nivel de conflicto.

También se reconoció que toda relación de interdependencia implicaba relaciones de poder entre los países participantes, las cuales podían ser asimétricas y complejas. De alguna forma se reconoció el papel que la política pública y la normatividad podían tener como instrumentos que influyesen en el carácter de la interdependencia, ya que, de acuerdo con Keohane y Nye (1989), ello influiría en la sensibiliidad y vulnerabilidad que cada país experimentaba. Un desarrollo posterior de estos conceptos se dio con Kroll (1993), quien discute que la sensibilidad como concepto se entenderá como algo ligado a la interdependencia; mientras que el concepto de vulnerabilidad se encuentra dentro del marco de análisis de la dependencia. Por tanto, los conceptos de cooperación y conflicto se verían desde esa perspectiva.

A pesar de que estos autores no ofrecieron mayor operacionalización o propuesta de medición de la interdependencia, fueron otros quienes desarrollaron varias propuestas al respecto. Otros autores también han estudiado los efectos que desencadena la interdependencia económica sobre el nivel de conflicto que se establece entre dos o más Estados-nación. Así, Fairlie y Queija (2007) examinan los efectos que el comercio tiene sobre dicha relación desde tres perspectivas: a) quienes consideran que el comercio promueve la paz; $b$ ) quienes consideran que el comercio tiene un reducido impacto en alentar o disuadir el conflicto entre los líderes y c) los neomarxistas, quienes sostienen que el comercio promovería la paz cuando las relaciones entre Estados son simétricas, en tanto que las relaciones asimétricas incrementan la posibilidad de conflicto. Barbieri (1996) analizaba las relaciones de comercio-conflicto o cooperación-conflicto de manera similar a las tres perspectivas mencionadas por Fairlie y Queija; no obstante, considera una perspectiva adicional: la idea de que el comercio es irrelevante al conflicto.

En suma, la interdependencia económica, analizada a través del comercio entre países, incluye (entre los elementos de su análisis) la idea de una relación comercial que pone en evidencia la vulnerabilidad del socio comercial, su sensibilidad para afrontar los cambios que se dan en el mercado internacional y todo ello influye en el establecimiento de relaciones simétricas o asimétricas que se traducen en un incremento o disminución de los conflictos bilaterales. 
Respecto de los trabajos empíricos que estudian las relaciones de interdependencia económica asociadas al conflicto, éstos se clasifican a la luz de las diferentes perspectivas ya mencionadas. En este apartado se presentan algunos de los enfoques teórico-metodológicos utilizados para la medición de la interdependencia económica entre varios países.

Uno de los primeros trabajos que ofrece una aproximación cuantitativa de las relaciones de interdependencia fue el de Hirschman (1945), quien analiza el aspecto político del comercio internacional y propone la elaboración de un índice de concentración del comercio, con el fin de identificar el peso de cada país en el comercio internacional y, con ello, observar el nivel de poder alcanzado en el contexto internacional. Las principales variables utilizadas para esa medición son las exportaciones e importaciones, a partir de las cuales se construye un índice de concentración del comercio internacional.

Mark Gasiorowski (1986) analiza también la relación existente entre la interdependencia económica y el conflicto internacional. El autor se concentra en indagar los efectos de las políticas internacionales en términos de interdependencia, al examinar de qué manera éstas afectan el nivel de conflicto entre países. Se examina si el clima de interdependencia tiene la capacidad de propiciar, en mayor o menor sentido, las relaciones de conflicto entre los países involucrados. Gasiorowski encuentra qué incrementos en las interacciones económicas (las cuales mide a través del comercio) están asociados con una disminución en los conflictos cuando algunos de los costosos resultados de estas interacciones son controlados. ${ }^{1}$

Desde la perspectiva de la historia económica, Katherine Barbieri (1996) sostiene que la interdependencia económica se relaciona con procesos de paz o conflicto, en los que los lazos comerciales cumplen el papel de facilitar la paz interestatal. Ella examina diversas relaciones bivalentes que se produjeron durante el periodo 18701938 y encuentra que los lazos económicos tienen una influencia importante en las disputas entre países con intervención militar, pero no en el caso de las guerras. En términos empíricos, Barbieri recurre al índice de Hirschman para la construcción de un índice de relevancia del comercio y un índice de simetría, con el fin de capturar el nivel de interdependencia entre países. De esta manera, por medio de un modelo multidimensional de interdependencia, con la inclusión de variables de control, se evalúa la relación existente entre interdependencia y conflicto. Destaca que la interdependencia extrema de tipo asimétrica (simétrica) tiende a incrementar (disminuir) los lazos de conflicto.

\footnotetext{
${ }^{1}$ La evidencia empírica del autor se sustenta en el estudio de indicadores de comercio (exportaciones, importaciones y balanza comercial), así como algunos indicadores que miden conflicto para ciento treinta países, durante el periodo 1948-1977, utilizando para ello una técnica de correlación, combinando la sección cruzada y series de datos nacionales.
} 
Harrison (1988) presenta su propuesta al aplicar la teoría moderna de la negociación vinculada con la teoría de economía política internacional. Este autor muestra que la interdependencia económica asimétrica no implica una negociación que ejerza influencia política sobre otros, por ende, para que el uso de la interdependencia económica se traduzca en influencia política, se requiere que el intercambio de recursos económicos, producto de concesiones políticas, permita a ambas partes el desarrollo de una mejor relación, ello en comparación con la relación que éstas hayan tenido a partir de la distribución de las ganancias que produce una relación económica única.

Un interesante trabajo que dio pie al presente artículo es el de Zhang (1995), quien presenta un análisis del potencial de la interdependencia económica para el desarrollo de una comunidad de seguridad en el conjunto de países que conforman la región Asia-Pacifico (China, Japón, Rusia y Estados Unidos). Para ello, Zhang estudia la relación entre interdependencia económica y la cohesión de una comunidad de seguridad, para el periodo 1979-1992, que se extendió hasta 1994. El autor plantea tres hipótesis centrales: a mayor interdependencia económica de los Estados: $a$ ) más fuerte es la actitud de cohesión de una comunidad de seguridad; $b$ ) más fuerte es el comportamiento de cohesión de una comunidad de seguridad; c) a mayor homogeneidad de las actitudes políticas, mayor coherencia en el comportamiento de los cuatro Estados en la región Asia-Pacífico. Zhang analiza la interdependencia económica a partir de cuatro indicadores comúnmente utilizados para analizar el flujo comercial entre los cuatro países analizados, así como indicadores de la IED.

En este mismo orden, Interdependent Task Force (2012) sugiere establecer una comunidad económica en Norteamérica como una estrategia para afrontar los cambios globales, ante un proceso inevitable e irreversible de la integración entre México, Estados Unidos y Canadá, debido a la evidente profundización de las relaciones que han desarrollado como socios comerciales (a partir de la firma del TLCAN), a la elaboración de una agenda en común sobre temas de seguridad, así como al hecho de que los gobiernos comparten principios comunes como democracias liberales.

Los trabajos relacionados con la estimación cuantitativa de la interdependencia a través del comercio tomaron como aproximación el análisis de los ciclos económicos, así como las redes globales de producción. En el caso mexicano, por ejemplo, la evidencia empírica en la que se muestra un incremento de la interdependencia se da en la frontera norte. Así, Philips y Cañas (2004) aplican un modelo dinámico de factores simples para medir el ciclo de negocios en cuatro áreas metropolitanas de la frontera de Texas y México. El objetivo es medir el grado de integración económica entre las ciudades de la frontera, usando un análisis de correlación, de cluster (conglomerado) y espectral. El índice del ciclo de negocios muestra que los cambios en la 
región de la frontera están fuertemente correlacionados con los cambios en el estado de Texas y con la economía de Estados Unidos. Los resultados sugieren que las áreas estudiadas están significativamente integradas con las economías abiertas.

Otro estudio de esta índole es el de Gordon Hanson (2001), quien examina varias ciudades de la frontera México-Estados Unidos en el periodo 1975-1977, con el objetivo de evaluar si la integración México-Estados Unidos ha contribuido a la expansión de la actividad económica en la región. De sus resultados se desprende que por un incremento del 10 por ciento de las exportaciones manufactureras de México, el empleo en las ciudades vecinas de la frontera sur de Estados Unidos incrementa del 1 al 2 por ciento. Igualmente subraya que la integración económica contribuye a una relocalización espacial de recursos, ayudando a explicar el patrón de soporte político para los acuerdos de comercio.

Finalmente, algunos autores afirman que México, Estados Unidos y Canadá se encuentran en el primer nivel del proceso de integración, es decir, un área de libre comercio (eliminación de tarifas y cuotas entre las partes); no obstante, el siguiente nivel, un mercado común, implicaría adicionalmente libre movimiento de personas y capital (Atkinson, 1998: 163).

Dentro de esta sinergia de integración, México tendría que enfrentar grandes retos, sobre todo porque llegar a ser parte de un mercado común le implicaría retos importantes, como que las exportaciones mexicanas tienen que recuperar, mantener, incrementar y consolidar su presencia en dichos mercados (Vega, 2010: 304).

Por lo tanto, el concepto de interdependencia económica resulta relevante para el caso de México y Estados Unidos, en cuanto a que ambos países comparten históricamente una relación económica, política y social, que va más allá del comercio bilateral. Sin embargo, estaríamos de acuerdo en destacar la necesidad de una medición bilateral del comercio, con el propósito de comprobar los niveles de esa interdependencia y validar (o no) la evidencia empírica anterior. La literatura teórica y empírica destaca el carácter de las relaciones de interdependencia como simétricas o asimétricas. En suma, con el análisis de los resultados cuantitativos se intenta revisar si se da una convergencia hacia la simetría y, con ello, una reducción de los conflictos, o si se mantiene o se incrementa una situación de asimetría. Dichos análisis se presentan en los apartados subsiguientes.

\section{ÍNDICE DE INTERDEPENDENCIA ECONÓMICA}

Aquí se retoman algunas de las perspectivas teórico-metodológicas abordadas con el objetivo de identificar y revisar el nivel y carácter de la interdependencia económica 
entre México y Estados Unidos, a partir de analizar las dimensiones del comercio bilateral entre dichos países, como la dirección del comercio, la interdependencia mutua y la asimetría. Así, en este artículo se retoman algunas de las propuestas de operacionalización de un índice de interdependencia planteadas por autores como Barbieri (1996) y Zhang (1995).

Una tarea inicial sería comprobar si existe una mutua dependencia bilateral en el ámbito comercial entre México y Estados Unidos, analizando los indicadores de exportaciones e importaciones totales. Por lo tanto, se intenta identificar la interdependencia económica entre ambos países, observando:

1) Los flujos recíprocos de importación y exportación de México y Estados Unidos.

2) La participación de las exportaciones e importaciones mexicanas con Estados Unidos respecto del total de éstas.

3) La cuantificación de la importancia de los productos mexicanos para el mercado de Estados Unidos.

El análisis se realiza mediante la construcción de cuatro indicadores propuestos en el trabajo de Zhang (1995); en este caso, se utiliza una adaptación de estos indicadores al análisis del comercio bilateral México-Estados Unidos. Estos índices son los que se proponen a continuación.

\section{La concentración geográfica del comercio}

De acuerdo con la teoría de la interdependencia propuesta por Zhang, un país que concentra su comercio exclusivamente con sólo un país es extremadamente sensible al desempeño económico del país al que exporta. Mientras que si un país mantiene su comercio exterior diversificado con diferentes países, se reducen los riesgos de dependencia y sensibilidad. En esa medida, se presentan las expresiones (1) y (2) como

$$
C E_{D}=\left.E_{D, F}\right|_{D T}
$$

donde el subíndice D significa “doméstico" y el subíndice F significa "foráneo" y $C E_{D}=$ La concentración geográfica de las exportaciones de México hacia Estados Unidos

$E_{D, F}=$ Exportaciones totales de México hacia Estados Unidos

$E T_{D}=$ Total de exportaciones de bienes de México

$$
C I_{D}=I_{D, F} / I T_{D}
$$


donde:

$C I_{D}=$ La concentración geográfica de las importaciones de México procedentes de Estados Unidos

$I_{D, F}=$ Importaciones totales de México procedentes de Estados Unidos

$I T_{D}=$ Total de importaciones de bienes de México.

Los coeficientes (1) y (2) propuestos, medidos en porcentajes, tienen un valor de 0 a 1 . Cuanto más se acerque al valor de 1 el coeficiente indica una mayor concentración geográfica del comercio de un país respecto de su socio bilateral. Asimismo, un mayor índice de concentración geográfica de las exportaciones muestra cómo los cambios en la actividad económica del país socio traen costosos cambios al primero de ellos y viceversa para el caso de las importaciones.

A partir del análisis de los flujos de comercio bilateral para el periodo 1980-2010, se estudia la tendencia de la participación del comercio de cada país sobre el tiempo, por lo tanto, es posible observar si una relación de comercio bilateral ha llegado a ser, en ambos casos, altamente dependiente (o sensitiva).

\section{La intensidad o nivel de dependencia del comercio bilateral}

La intensidad comercial bilateral mide la importancia de los productos exportados o importados en el mercado del país socio. En ese sentido, este indicador no sólo mide el flujo de comercio de dos vías, sino, además, cuantifica la importancia de los bienes comerciados para el mercado del país socio. Para efectos de este trabajo, y de acuerdo con la perspectiva teórica de interdependencia que manejan tanto Keohane y Nye (1989) como Zhang (1995), ${ }^{2}$ este indicador es nuestra medida de dependencia de la demanda de mercado del país con el cual se comercia.

$$
I E_{\text {México }}=\left(E_{D, F} / I T_{F}\right) /\left(E_{D, F} /_{E T_{D}}\right)
$$

donde:

$I E_{D}=$ Índice de dependencia de las exportaciones de México respecto de Estados Unidos

$E_{D, F}=$ Exportaciones de bienes de México a Estados Unidos

\footnotetext{
2 Para la elaboración de los índices de interdependencia que se construyen en este trabajo, nos basamos en el desarrollo teórico-metodológico de Zhang (1995: 199-201).
} 
$I T_{F}=$ Total de importaciones mundiales de bienes de Estados Unidos

$E T_{D}=$ Total de exportaciones de bienes de México.

$$
I I D=\left(I_{D, F} /_{E T_{F}}\right) /\left(I_{D, F} / T_{D}\right)
$$

donde:

$I I_{D}=$ Índice de dependencia de las importaciones del comercio bilateral MéxicoEstados Unidos

$I_{D, F}=$ Importaciones de México procedentes de Estados Unidos

$E T_{F}=$ Total de las exportaciones de bienes de Estados Unidos

$I T_{D}=$ Importaciones totales de bienes de México.

A diferencia de los coeficientes de concentración geográfica, los índices tomarían valores mayores a 1. Los indicadores presentados en líneas anteriores se adaptaron para el análisis bilateral de la interdependencia económica entre México y Estados Unidos.

En la siguiente sección, se analizan los resultados de los índices propuestos, con el objetivo de comprobar nuestra hipótesis de interdependencia.

\section{EVIDENCIA EMPÍRICA DE LA INTERDEPENDENCIA ECONÓMICA MÉXICO-Estados Unidos}

En este apartado se analizan los resultados de los índices propuestos en la sección anterior, para evidenciar el nivel de interdependencia económica que existe entre ambos países. Asimismo, se incluye en el análisis la IED, considerada como transacciones internacionales que han resultado significativas para México, cuyo origen es principalmente de Estados Unidos. Asimismo, la frontera norte de México y la frontera sur de Estados Unidos es la región donde fue más evidente esta relación de interdependencia y, por ello, se incluyen datos sobre la relación comercial en dicha región.

Como lo apunta Humberto Garza (1996), la relación económica comercial entre ambos países se basa en las características propias derivadas de la cercanía geográfica que comparten y que estimula la complementariedad de sus economías, sus disputas territoriales pasadas, así como los contextos sociales y políticos que estimulan la existencia de intensas interacciones culturales, de personas, bienes, etc. De ahí que sea oportuno un examen de la relación de interdependencia, a partir de los diferentes coeficientes de esta relación comercial bilateral. 


\section{Concentración geográfica de las exportaciones e importancia de los productos mexicanos en el mercado estadunidense}

En este apartado se presenta un análisis de la evolución de los flujos comerciales que intentan mostrar la interdependencia económica entre México y Estados Unidos. Una particularidad en la relación es su vecindad geográfica, lo cual impone una característica particular a las relaciones de dependencia entre ambos países. El hecho de que compartan límites territoriales y que éstos se encuentren ligados a un contexto histórico de lucha por los mismos, determina la necesidad de que ambos países interactúen, ${ }^{3}$ y explica, a la vez, la relación de dependencia asimétrica que han establecido a partir del comercio internacional.

Por otro lado, se deben destacar las transformaciones que sufre el sector exportador y que marcan la dinámica de la relación comercial con Estados Unidos. ${ }^{4}$ Entre las principales características de dichas transformaciones se halla el cambio en la composición de las exportaciones (las manufactureras representaron el 32 por ciento en el total exportado de 1980; mientras que en 1998 dichas exportaciones significaron el 89.8 por ciento del total), el crecimiento de la división maquinaria y equipo en el total exportado (el 22 por ciento en 1980 y el 70 por ciento en 1998), la participación de las exportaciones maquiladoras (el 14 por ciento en 1980 y el 45 por ciento en 1998), así como el crecimiento del contenido importado en las exportaciones manufactureras (el 40 por ciento en 1993 a casi el 50 por ciento en 2005). ${ }^{5}$

La gráfica 1 muestra los resultados para el coeficiente de concentración geográfica de exportaciones $\left(C E_{D}\right)$ y el índice de dependencia de las importaciones de Estados Unidos de productos mexicanos $\left(I E_{D}\right)$ para el periodo 1980-2011.

Se presenta en una sola gráfica $C E_{D}$ e $I E_{D}$ para evidenciar un proceso contrario de ambas variables. Mientras que para México el mercado de Estados Unidos es el más importante para sus exportaciones, la intensidad o dependencia de Estados Unidos por productos mexicanos en su mercado doméstico es reducida y ha disminuido. El coeficiente $C E_{D}$ indica que la participación de las exportaciones de México dirigidas al mercado de Estados Unidos en el total de sus exportaciones pasó de 0.53 en 1981 a índices cercanos a 0.9 durante la segunda mitad de los noventa (véase la gráfica 1). En la gráfica 1 se observa que el incremento en el índice más importante se

\footnotetext{
${ }^{3}$ Una interacción que no sólo se da en el aspecto económico, pues trata problemas en común, por ejemplo, la migración, el tráfico de drogas, los temas del medio ambiente, entre otros.

${ }^{4}$ Varios estudios sobre el proceso de liberalización económica emprendido durante estos años dan cuenta de la transformación del sector externo manufacturero (Casar, 1989; Aspe, 1993; Lustig, 1995; Arjona y Unger, 1996; Villarreal, 1997).

${ }^{5}$ Para nuestro estudio, la desagregación de las importaciones por tipo de bien, se empieza en 1993 y se concluye hasta junio de 2006, conforme a los datos proporcionados por el INEGI.
} 
produce antes de la firma del TLCAN; mientras que a partir de la integración comercial con este país se observa un comportamiento más estable del índice $C E_{D}$.

\section{Gráfica 1}

CONCENTRACIÓN DE EXPORTACIONES A ESTADOS UNIDOS

E ÍNDICE DE INTENSIDAD DE IMPORTACIONES DE ESTADOS UNIDOS

DE PRODUCTOS MEXICANOS

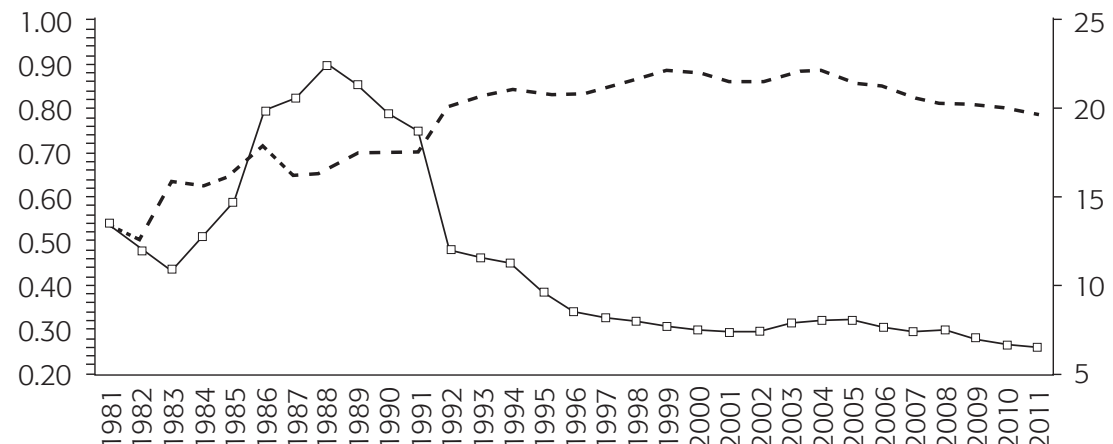

$$
--C E_{D} \longrightarrow-I E_{D}
$$

Fuente: Elaboración propia, con información de United Nations, Commodity Trade Statistics.

Si bien aquí sólo se incluyen los totales exportados, habría que añadir que la estructura de las exportaciones cambió a partir del proceso de apertura comercial que se inició a mediados de los ochenta, cuando la política industrial dirigida a un incremento de las exportaciones manufactureras dio los resultados esperados con un aumento de las exportaciones manufactureras en el total exportado (el 30 por ciento en 1980; el 90 por ciento en 1999 y el 82 por ciento en 2010), mientras que las exportaciones de bienes primarios como la petrolera redujeron su participación (el 58 por ciento en 1980 y el 6 por ciento en 1998), hasta en estos últimos años cuando se recuperó de nuevo su porcentaje, debido a un incremento de los precios internacionales del petróleo (el 14 por ciento en 2010). Más que la integración comercial con Estados Unidos, ha sido evidente que los flujos de exportaciones dirigidas al mercado estadunidense en gran medida se explican por el proceso de liberalización comercial de finales de los ochenta, la flexibilización de la Ley de Inversión Extranjera Directa, la política cambiaria, arancelaria y por el peso de la actividad maquiladora en el proceso exportador (Barajas, 2000; Villareal, 2010); la evidencia empírica demuestra que fueron las políticas de liberalización económica de finales de los ochenta las que sentaron 
las bases para el crecimiento del sector exportador, teniendo la firma del TLCAN como la culminación de ese proceso. ${ }^{6}$

Por otro lado, la estrecha relación establecida entre la economía de México y la de Estados Unidos vuelve al primero más vulnerable a los acontecimientos y problemas del desarrollo de su socio comercial. Muestra de ello han sido las diferentes crisis económicas que ha confrontado Estados Unidos, por ejemplo, la de 2001 y, en particular, desde 2007. Con la crisis económica de 2001, el principal impacto se dio a partir de la reducción de las exportaciones del sector maquilador y del número de empleos que producía dicho sector (Cañas et al., 2004; Gruben, 2004). En tanto, la crisis que inició entre 2007 y 2008 ha tenido un impacto más expansivo en la actividad económica de Estados Unidos. Una de las respuestas a la concentración geográfica de las exportaciones en el mercado estadunidense ha sido la búsqueda de nuevos mercados, pues a partir de esta crisis ya tan sólo el 78.8 por ciento de las exportaciones de México se destinan al mercado de Estados Unidos, lo que representa una disminución del 9.6 por ciento respecto de lo exportado en $1998 .^{7}$ Cabe mencionar que México ha buscado diversificar su mercado de exportaciones hacia regiones que serían potencialmente importantes, como el mercado latinoamericano, europeo y asiático. En esta búsqueda, México ha llegado a ser una de las economías más abiertas en el mundo, con mayor cantidad de tratados comerciales firmados. ${ }^{8}$

Como consecuencia de las relaciones de dependencia con su país vecino, las decisiones de política exterior de México se encuentran limitadas, pues las condiciones económicas del país no permiten que exista simetría en la relación económica con Estados Unidos. Esta condición asimétrica se observa en los términos del TLCAN y en los acuerdos y pactos firmados entre ambos países, cuyos términos y condiciones claramente favorecen (directa o indirectamente) a Estados Unidos (Garza, 1996; Vega, 2010).

La gráfica 1 muestra, además, los resultados para el índice de dependencia de las importaciones de Estados Unidos por productos mexicanos $\left(I E_{D}\right)$. Como se advierte, los productos mexicanos que van al mercado estadunidense no son significativos en el total del comercio exterior de dicho país. Aún más, el índice construido muestra un crecimiento del coeficiente para el periodo 1983-1986, y de ahí una caída hasta 1992, lo cual se explica más por los cambios en el denominador del coeficiente (siendo las exportaciones del sector de maquinaria y material de transporte las que dan un salto importante en esos periodos). De tal manera, se concluiría que en la

\footnotetext{
${ }^{6}$ Entre los trabajos que presentan una estimación de tipo cuantitativa del impacto de la liberación económica sobre las exportaciones se hallan Casar (1989), OCDE (1996) y Villarreal (1997).

${ }^{7}$ Una revisión de las exportaciones por país de destino mostrará que a partir de la entrada de China a la OMC, las exportaciones mexicanas hacia el país asiático se han ido incrementando (véase $<$ www.economia.gob.mx $>$ ).

${ }^{8}$ A la fecha, México tiene firmados doce tratados de libre comercio (véase $<$ www.economia.gob.mx $>$ ) con varios países del mundo.
} 
mayor parte del periodo estudiado los productos mexicanos no representan, a nivel agregado, un vínculo de dependencia de Estados Unidos hacia México.

En síntesis, si bien el acuerdo comercial significó la apertura del mercado interno estadunidense para la producción mexicana, México no ha destacado en términos de su importancia respecto del total importado por ese país, como sería lo esperado con la firma del TLCAN. Una de las variables que explican este desempeño a partir de 2001 ha sido la entrada de China a la OMC. ${ }^{9}$ Cuando entra en vigor el TLCAN, China aún no se perfilaba como una competencia real para los productos mexicanos en el mercado estadunidense; no obstante, con la entrada de China en la OMC, sectores como el textil se vieron negativamente afectados por la competencia de los productos chinos en este mercado (Hanson y Robertson, 2008; Fonseca, 2010).

Los resultados hasta aquí presentados muestran claramente cómo se ha configurado una relación de interdependencia económica asimétrica entre ambos países y cómo dicha asimetría se ha acrecentado conforme México se integra al comercio en Norteamérica. Mientras que la economía mexicana tuvo como objetivo reestructurarse alrededor del sector exportador, el mercado estadunidense resultó significativo para un crecimiento económico basado en las exportaciones. En contraste, la gráfica 1 también muestra que no ocurre lo mismo para la economía de Estados Unidos, ya que los índices construidos destacan que México tuvo una participación poco significativa en las importaciones estadunidenses que apenas alcanzaron los siete puntos en 2011.

Si bien los índices aquí propuestos enfatizan esta asimetría en los flujos de exportaciones de ambos países, es necesario desagregar la información a nivel regional donde se podrá apreciar que los flujos comerciales provenientes de México son sensiblemente significativos para los estados de la frontera sur de Estados Unidos, situación que se analizará en secciones posteriores. Enseguida se procede a la explicación de los resultados obtenidos en la construcción de un coeficiente de concentración geográfica para las importaciones y de dependencia de las exportaciones procedentes del mercado estadunidense y dirigido al mercado mexicano.

\section{Concentración geográfica de las importaciones y dependencia de las exportaciones procedentes del mercado estadunidense hacia México}

Antes de proceder al análisis de los coeficientes de importación, se hace necesario un contexto de introducción a las transformaciones en el sector que ayudaran a la expli-

\footnotetext{
${ }^{9}$ En términos de las importaciones, China pasó de ser el decimoquinto socio comercial de Estados Unidos en 1989, a ser el tercer socio (después de México) (International Trade Administration, 2012).
} 
cación de los coeficientes. Como se ha mencionado, durante la apertura comercial las importaciones procedentes de Estados Unidos debían servir para sustentar el modelo económico basado en la exportación de bienes manufactureros. De tal forma que, durante los periodos de 1981, 1990, 2000 y 2010, los principales productos exportados por Estados Unidos hacia México fueron predominantemente productos manufacturados y de capital, seguidos de bienes manufacturados y en menor medida de productos tales como los combustibles minerales, entre los cuales se encuentra el petróleo. Aún más, las importaciones de bienes de capital y, en mayor medida, de los bienes intermedios, se dirigen a las actividades de exportación. En la gráfica 2 se presentan los datos para el periodo 1993-2006 sobre el desempeño de los bienes intermedios destinados a la actividad de exportación, esto como razón de las exportaciones totales, así como del total de las importaciones de bienes intermedios. ${ }^{10}$

\section{Gráfica 2}

IMPORTACIONES DE BIENES INTERMEDIOS ASOCIADAS A LAS EXPORTACIONES

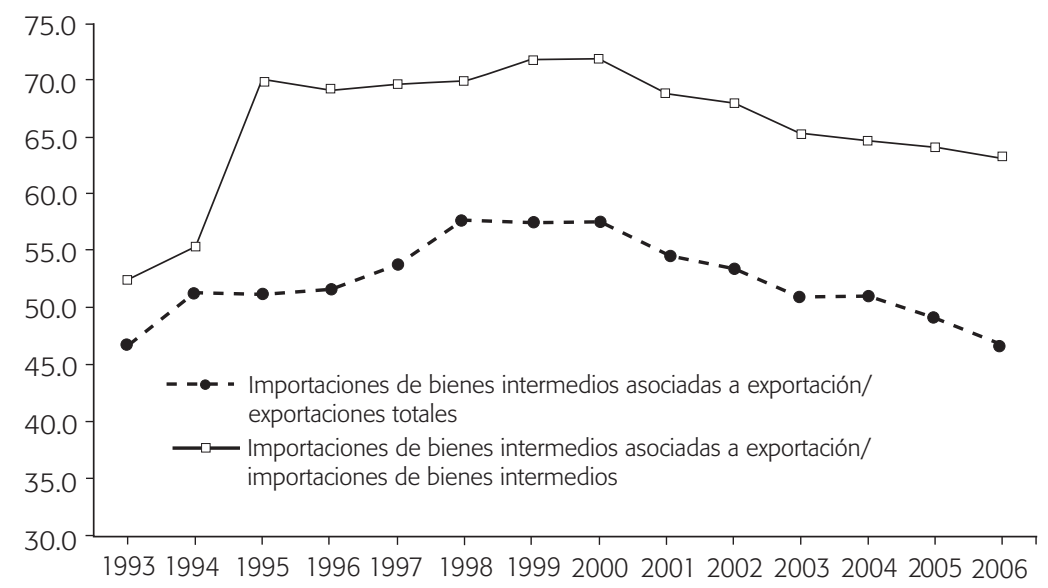

Fuente: Elaboración propia, con información de United Nations, Commodity Trade Statistics.

La gráfica 2 muestra cómo las importaciones de bienes intermedios desde Estados Unidos representaron más del 50 por ciento del total exportado de dicho país para la mayor parte del periodo. Durante los años de la crisis económica del 2000-2002

${ }^{10}$ Los datos provienen del Anexo Estadístico de los Informes de Gobierno. No se encuentran cifras desagregadas posteriores al año 2006. Si bien están generadas por el Banco de México, ya no forman parte de sus estadísticas publicadas. 
en Estados Unidos, se produjo una reducción en los flujos de comercio con este país. ${ }^{11}$ No obstante, no es posible negar que en el periodo al que se refiere este estudio se importaba para sostener el modelo económico basado en las exportaciones manufactureras, así como para cumplir con el papel asignado en las redes globales de producción. De igual manera, lo muestra la razón entre las importaciones de bienes intermedios asociados a la exportación como porcentaje del total de bienes intermedios importados. En promedio, para el periodo 1993-2006 éstas constituyen entre el 65 y el 70 por ciento del total de bienes intermedios. ${ }^{12}$

Luego de la recuperación económica, las importaciones siguieron cumpliendo su papel de soporte a la exportación de productos manufactureros, mientras que la reducción gradual de aranceles que el calendario del TLCAN marcaba, permitía la entrada de un mayor número de bienes al mercado mexicano, intensificándose de esta forma la concentración geográfica de las importaciones con este país.

La gráfica 3 muestra los resultados para los índices de concentración geográfica de las importaciones $\left(\mathrm{CI}_{D}\right)$ y dependencia de las importaciones procedentes de Estados Unidos hacia el mercado mexicano $\left(I I_{D}\right)$. Con estos índices, se evidencia que las importaciones procedentes de Estados Unidos resultan altamente significativas para el mercado de México, así como para su producción dirigida a la exportación.

Si bien la gráfica 3 refleja cómo el coeficiente de concentración geográfica $C I_{D}$ ha disminuido significativamente a partir de 2001, no es posible deducir que lo que se produce es una diversificación de las importaciones procedentes de terceros países, ya que de acuerdo al índice $I I_{D}$ (gráfica 3 ), se destaca que los productos del mercado de Estados Unidos han aumentado su importancia en el mercado mexicano. Se reitera que un elemento clave en la evolución de dichas importaciones ha sido la entrada de China a la omc en 2001 (Fonseca, 2010), periodo en que las exportaciones tanto de México hacia Estados Unidos y viceversa empezaron a tener una menor participación respecto de las exportaciones totales de ambos países, ya que de inmediato el mercado asiático fue atractivo para las filiales de los grandes corporativos.

\footnotetext{
${ }^{11}$ Entre las referencias sobre los efectos de esta crisis en la economía mexicana, en particular para sectores que se dirigen a la exportación como la maquiladora, véanse Acevedo (2002), Mundra y Gerber (2010) y Pérez (2006).

${ }^{12}$ De hecho, se generó un saldo negativo en la cuenta corriente, aunado a un tipo de cambio fijo insostenible a finales de 1994, cuando se procedió a la devaluación del peso mexicano.
} 


\section{Gráfica 3}

CONCENTRACIÓN DE IMPORTACIONES DE ESTADOS UNIDOS

E ÍNDICE DE DEPENDENCIA DE EXPORTACIONES DE ESTADOS UNIDOS A MÉXICO

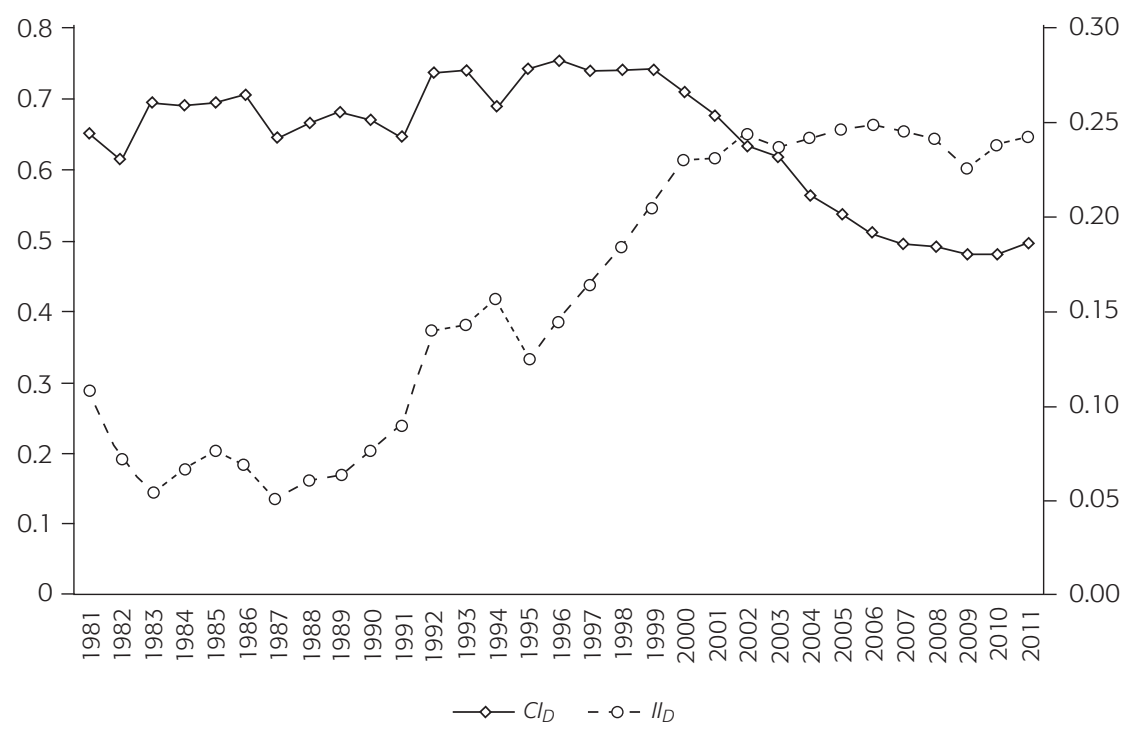

Fuente: Elaboración propia con información de United Nations, Commodity Trade Statistics.

Asimismo, la política de China en la economía global de hands-on (como la devaluación de su moneda), contraria a la de México de hands-off, trajo como consecuencia la pérdida para México de una porción significativa del mercado estadunidense, de tal forma que a partir de 2007 China desplazó a México como segundo socio comercial (Hernández, 2012). Con ello se produjo un efecto de sensibilidad indirecta en su relación de interdependencia con Estados Unidos.

El incremento en la competencia por el mercado mexicano no ha sido suficiente para restar la importancia de los productos estadunidenses hacia el mercado mexicano. Prueba de ello se muestra en la gráfica 3, al observar que el coeficiente $I I_{D}$ aún tiene un valor de 0.50 hacia 2011, a diferencia del coeficiente $I E_{D}$, en el que se evidencia la presencia de comportamientos contrarios, cuyo resultado es el valor del índice que se va reduciendo para el periodo estudiado. En el caso de $I I_{D^{\prime}}$ se presentan dos comportamientos que se complementan para resultar en un valor del índice que se incrementó durante el periodo estudiado y que permanece más o menos estable a partir de 2001.

En el caso de la dependencia de las importaciones de productos estadunidenses, se presentan dos situaciones: 1) se tiene un incremento de los flujos de bienes de 
Estados Unidos hacia México respecto del total de lo enviado al resto de los países y, 2) se presenta un incremento de la participación de las importaciones de estos bienes procedentes de Estados Unidos respecto del total importado por México; en conjunto, se tiene como resultado una marcada dependencia de las importaciones de productos estadunidenses.

Los índices hasta aquí presentados han contribuido a explicar que la relación de interdependencia económica es compleja en varios sentidos: en primer lugar, resalta la posición de vulnerabilidad de la demanda por las exportaciones mexicanas y la relativa importancia de los productos mexicanos en el mercado de Estados Unidos. En segundo, si bien el coeficiente de concentración geográfica indica que México ha reducido la participación de las importaciones procedentes de Estados Unidos, el índice de dependencia indica que la importancia de los productos importados para el mercado mexicano se ha mantenido en los últimos años, todo lo cual da por resultado una situación de interdependencia asimétrica que existe entre ambos países y que se sostiene y no se ha resuelto después de la firma del TLCAN.

Con el fin de complementar el análisis presentado, se incluye un análisis sobre el comportamiento de los flujos comerciales en la región fronteriza que comparten ambos países.

\section{Exportaciones e importaciones de los estados de la frontera sur estadunidense a México}

Como se ya se indicó, una de las contribuciones de este trabajo es incluir, además de la cuantificación de los índices de interdependencia, las variables complementarias que nos permitan realizar un análisis más íntegro de la relación de interdependencia entre México y Estados Unidos. En este apartado se concentra el análisis por regiones, donde se destaca la frontera norte de México y los estados de la frontera sur de Estados Unidos, los cuales representan para ambos países una participación importante en el total comerciado, tanto en términos de manufacturas como de servicios. La relación comercial de las regiones fronterizas México-Estados Unidos da cuenta de las grandes diferencias entre ambos países, a pesar de las cuales existe una larga lista de momentos de cooperación o conflicto en torno a las problemáticas en común, incluyendo las derivadas de la relación comercial. Asimismo, Hanson (1996) encuentra que la dinámica de las exportaciones manufactureras de la frontera norte ha sido significativa en la generación de empleos en las ciudades de la frontera sur de Estados Unidos.

$\mathrm{Al}$ analizar la estructura de las exportaciones por estado de la frontera sur de Estados Unidos en datos relativos, cabe observar a México como principal mercado 
de los productos de los cuatro estados de la frontera sur de Estados Unidos, ejemplo de ello es que, en promedio para el periodo 1999-2011, el 60 por ciento del total exportado a México por Estados Unidos corresponde a estos cuatro estados de la frontera.

\begin{tabular}{|lcccc|}
\hline \multicolumn{4}{c|}{ Cuadro 1 } \\
& \multicolumn{4}{c|}{ FLUJOS COMERCIALES DE LOS ESTADOS DE LA FRONTERA } \\
& SUR ESTADUNIDENSE HACIA MÉXICO \\
Porcentaje de exportaciones & Porcentaje de importaciones \\
Estado & 2000 & 2011 & 2000 & 2011 \\
Estados Unidos & 100 & 100 & 100 & 100 \\
Texas & 43.64 & 44.26 & 42.75 & 44.10 \\
California & 15.63 & 13.07 & 15.68 & 13 \\
Arizona & 4.18 & 3.02 & 4.16 & 3 \\
Nuevo México & 0.11 & 0.24 & 0.11 & 0.20 \\
Otros & 36.45 & 39.41 & 40.95 & 44.20 \\
Fuente: Elaboración propia, con información de International Trade Administration (2012). & \\
\hline
\end{tabular}

El cuadro 1 muestra los porcentajes de la balanza comercial para dos años representativos: 2000 y 2011, tanto de las exportaciones como de las importaciones de los estados fronterizos del sur de Estados Unidos. Como se advierte, la participación por entidad no ha variado significativamente en estos dos años. En el mismo cuadro 1 se observa que el principal exportador es el estado de Texas. A nivel sectorial, el comercio entre Texas y México es de carácter intraindustrial para el caso de computadoras y equipo electrónico (el 22.52 por ciento en términos de las exportaciones y el 26.56 por ciento, importaciones). Aun cuando lo anterior indicaría un comercio entre países de similar desarrollo, un análisis de los datos a nivel de mayor desagregación daría cuenta de un comercio intraindustrial de corte vertical. ${ }^{13}$ En general, conviene destacar que el comercio de dos vías se establece en mayor medida con California y Texas, lo cual significa que su proceso de integración comercial es más dinámico, en particular para el comercio de computadoras y productos electrónicos, maquinaria de transporte, equipo electrónico, plástico y derivados. México y la frontera norte, en especial, son destinos importantes de centros de ensamble de grandes multinacionales vinculadas a redes globales de producción, las cuales importan

${ }^{13}$ El comercio intraindustrial de carácter vertical indica que se da un comercio de bienes de diferente calidad o lo que también se conoce como un comercio norte-sur (Sotomayor, 2008). 
temporalmente maquinaria y equipo para después exportarlo al país de origen, sin que esto signifique que en México se realiza el total de las etapas del proceso de producción (Barajas, 2000).

En el plano regional, los principales estados importadores de mercancías mexicanas hacia Estados Unidos son Texas, California, Michigan y Arizona, que adquieren más del 60 por ciento de las exportaciones que México realiza a este mercado. En particular, Texas ha incrementado la participación de sus importaciones, siendo la vía terrestre el principal medio para el intercambio; por otra parte, aunque Michigan no es un estado fronterizo, sus importaciones destacan por la producción de autopartes. Otro elemento destacado es que California y Arizona han disminuido su participación en la importación de productos provenientes de México, lo cual se observa en el cuadro 2 .

\section{Cuadro 2}

EXPORTACIONES E IMPORTACIONES EN LOS ESTADOS FRONTERIZOS

\section{Porcentaje de exportaciones}

\begin{tabular}{|c|c|c|c|c|c|c|c|}
\hline Destino & Arizona & Destino & California & Destino & $\begin{array}{l}\text { Nuevo } \\
\text { México }\end{array}$ & Destino & Texas \\
\hline Mundo & 100 & Mundo & 100 & Mundo & 100 & Mundo & 100 \\
\hline México & 33.56 & México & 16.22 & México & 22.20 & México & 34.82 \\
\hline Canadá & 12 & Canadá & 10.85 & Israel & 21.15 & Canadá & 8.81 \\
\hline China & 5.64 & China & 8.92 & Canadá & 16.84 & China & 4.36 \\
\hline Japón & 4.70 & Japón & 8.23 & China & 4.29 & Brasil & 4.01 \\
\hline $\begin{array}{l}\text { Reino } \\
\text { Unido }\end{array}$ & 4.46 & Corea del Sur & 5.30 & Otros & 35.52 & Holanda & 3.50 \\
\hline Alemania & 4.33 & Hong Kong & 4.82 & & & Corea del Sur & 2.96 \\
\hline Otros & 35.31 & Otros & 45.67 & & & Otros & 41.54 \\
\hline \multicolumn{8}{|c|}{ Porcentaje de importaciones } \\
\hline Mundo & 100 & Mundo & 100 & Mundo & 100 & Mundo & 100 \\
\hline México & 35.10 & China & 34.16 & China & 32.84 & México & 29.04 \\
\hline China & 13.86 & Japón & 11.33 & Canadá & 15.43 & China & 11.40 \\
\hline Japón & 9.74 & México & 9.57 & México & 13.71 & Venezuela & 5.79 \\
\hline Canadá & 7.83 & Canadá & 5.83 & Japón & 7.98 & Arabia Saudita & 4.94 \\
\hline Malasia & 6.60 & Corea del Sur & 3.35 & Holanda & 6.57 & Canadá & 4.67 \\
\hline Otros & 26.87 & Otros & 35.75 & Otros & 23.48 & Otros & 44.15 \\
\hline
\end{tabular}

Fuente: Elaboración propia con información obtenida de International Trade Administration (2012). 
Por otra parte, del total de las importaciones de cada estado de la frontera sur estadunidense, sólo Texas (el 29.04 por ciento) y Arizona (el 35.10 por ciento) guardan una estrecha relación comercial con México; en tanto, para los estados de California y Nuevo México, China es el principal socio comercial, y en el caso de estos últimos, México ocupa el tercer lugar como país-origen de sus importaciones. Cabe subrayar que México es el principal destino de las exportaciones de tales estados, lo que es entendible por su localización geográfica y la ventaja comparativa que significa la cercanía geográfica entre estados fronterizos de ambos países. A pesar de esta ventaja comparativa, que se observa en el cuadro 2 , se encuentra que no es la misma dinámica a la inversa, pues México es sólo el principal origen de mercancías importadas para Texas y Arizona; mientras que para estados como California y Nuevo México los productos mexicanos ocupan ya no el primero, sino el tercer lugar de país-origen de los productos importados, siendo China, Japón y Canadá los principales mercados de origen.

También, resulta importante analizar cuál es el contenido del comercio en el ámbito fronterizo, encontrándose evidencias de especialización productiva. Por ejemplo, estados como Arizona importan principalmente productos agrícolas (el 34.34 por ciento), pero también resulta significativa la importación de equipo de transporte (el 15.8 por ciento); en el caso de Nuevo México, se observa que, del total de las importaciones procedentes de México, el 30.32 por ciento se concentran en equipo de transporte; en tanto, el 21.8 por ciento corresponde a productos animales. Cabe observar que, en tanto las exportaciones de los estados de la frontera sur de Estados Unidos a México son diversificadas, no existe el mismo efecto respecto de las importaciones procedentes de este segundo país. ${ }^{14}$ Aunque ambos países se han esforzado por minimizar sus diferencias o conflictos (derivados de imposiciones por barreras no arancelarias), con la finalidad de continuar con la vigencia del TLCAN, en su momento se ha puesto en evidencia la asimetría de la relación, pues, en términos del contrato, el TLCAN no considera esos elementos (como la movilidad de la fuerza laboral o el libre flujo de personas), y ha impuesto obstáculos a la libre movilidad del transporte de carga.

Además de todo ello, ambos países tienen ante sí un reto importante: facilitar los movimientos de bienes, servicios y personas a lo largo de la frontera, así como detener el trasiego de drogas y ordenar el flujo migratorio. En ese sentido, las políticas que se apliquen serán significativas (Turner y Díaz, 2009) para promover la coopera-

\footnotetext{
${ }^{14}$ Cabe reconocer que después de la entrada en vigor del TLCAN (en 1994), tanto México como Estados Unidos han enfrentado controversias relacionadas con el comercio, entre las que se hallan las barreras a la entrada de productos como los aguacates mexicanos, atún, carne porcina, harina de trigo, petróleo y, recientemente, el jitomate. A ello se deben agregar temas como la libre circulación del transporte mexicano dentro del territorio estadunidense y los temas de liberación de precios del azúcar en México (USMCOC, 2012).
} 
ción, que es consistente con una ampliación de la interdependencia entre ambos países, esto con menor asimetría.

\section{Inversión extranjera directa}

La IED es considerada otra variable que explica la relación de interdependencia bilateral, en la medida en que promueve un aumento de los flujos de comercio entre ambos países, pero también es una muestra de la asimetría que impone dicha variable. En este apartado se pone especial atención a los flujos de IED que se dirigen a las actividades de transformación y las que están ligadas a la producción para la exportación. En el caso de México, la IED fue evidente por la importancia de la actividad maquiladora en el total del comercio entre nuestro país y Estados Unidos. ${ }^{15}$ Mientras que la liberalización económica emprendida a fines de los ochenta sentó las bases para un ambiente favorable al capital extranjero, el TLCAN lo hizo de forma particular al incrementar los flujos de inversión dirigidos a los sectores productivos de transformación y, en buena medida, para la actividad maquiladora. El cuadro 3 muestra la serie de datos para los años en los que se tiene la desagregación respecto de la IED, la cual se dirige a la maquiladora. ${ }^{16}$

Entre las principales características de la IED que se recibe de Estados Unidos destaca su alto porcentaje de participación respecto del total nacional (véase el cuadro 3), ya que cerca del 60 por ciento de la IED provino de aquel país. En virtud de que se tienen los datos desagregados a nivel de la maquiladora, se diría que la IED de Estados Unidos ha sido significativa para este sector, incluso cabe señalar que los ciclos económicos se reflejan en estos porcentajes, puesto que la IED destinada a la maquiladora disminuyó en 2001 (de 2000 a 2001, del 20 al 8.7 por ciento), año en que hubo una caída en la actividad económica de Estados Unidos. La industria maquiladora ha sido, en general, para el periodo 1994-2006, receptora de casi la tercera parte del total de la IED. Una explicación de este desempeño radica en su concentración en las actividades relacionadas con la industria automotriz (productos metálicos, maquinaria y equipo), que tradicionalmente es la que ha establecido más partes y componentes de la producción en México.

${ }^{15}$ Las exportaciones del sector manufacturero (vía maquiladoras) constituyeron cerca de la mitad del total de bienes exportados hacia Estados Unidos, esto a mediados de los noventa; mientras que porcentajes similares se encuentran también para las importaciones manufactureras (Sotomayor, 2008). Ciertamente se suscitó un comercio intrafirma entre las filiales establecidas en el país receptor y la casa matriz, que se corresponde con una integración vertical de la producción.

${ }^{16}$ Como ya se dijo antes, el INEGI dejó de registrar las operaciones dirigidas a la actividad maquiladora hasta 2006. 


\begin{tabular}{|c|c|c|c|c|c|}
\hline \multicolumn{6}{|c|}{$\begin{array}{c}\text { Cuadro } 3 \\
\text { PORCENTAJES DE IED 1994-2006 }\end{array}$} \\
\hline Año & $\begin{array}{l}\text { IED de } \\
\text { Estados } \\
\text { Unidos }\end{array}$ & $\begin{array}{l}\text { IED de Estados } \\
\text { Unidos en } \\
\text { maquila }\end{array}$ & $\begin{array}{l}\text { IED maquiladora/ } \\
\text { manufacturero }\end{array}$ & $\begin{array}{l}\text { Frontera } \\
\text { norte/total } \\
\text { nacional }\end{array}$ & $\begin{array}{l}\text { Frontera } \\
\text { norte/IED } \\
\text { maquiladora }\end{array}$ \\
\hline 1994 & 46.73 & 16.6 & 14.42 & 19.18 & 40.14 \\
\hline 1995 & 65.79 & 21.71 & 28.15 & 29.27 & 51.34 \\
\hline 1996 & 66.97 & 23.5 & 29.85 & 24.38 & 68.46 \\
\hline 1997 & 61.42 & 18.73 & 23 & 33.59 & 36.97 \\
\hline 1998 & 65.08 & 35.35 & 40.97 & 31.66 & 67.73 \\
\hline 1999 & 54.04 & 32.59 & 30.27 & 30.71 & 50.44 \\
\hline 2000 & 71.79 & 19.87 & 29.85 & 31.42 & 42.28 \\
\hline 2001 & 71.8 & 8.72 & 36.56 & 14.94 & 41.87 \\
\hline 2002 & 55.23 & 13.79 & 23.5 & 19 & 38.74 \\
\hline 2003 & 54.61 & 20.67 & 26.7 & 25.01 & 43.22 \\
\hline 2004 & 36.85 & 24.05 & 17.87 & 15.59 & 56.05 \\
\hline 2005 & 47.89 & 22.14 & 25.66 & 34.08 & 29.7 \\
\hline 2006 & 64.22 & 20.68 & 29.83 & 28.19 & 46.66 \\
\hline $\begin{array}{l}\text { Promedio } \\
1994-2006\end{array}$ & 58.65 & 21.42 & 27.43 & 25.93 & 47.2 \\
\hline
\end{tabular}

En el marco del análisis de la IED por regiones, su evolución en la frontera norte merece especial atención, pues en gran medida el crecimiento económico se ha generado a partir de la puesta en marcha del programa maquilador, ello con el fin de estimular la industrialización en esa región. Cabe decir que esta iniciativa de México tuvo como respuesta el esquema tarifario de Estados Unidos conocido como 806 / 807, con el que se permitió que bienes estadunidenses exportados a México fuesen sujetos únicamente de impuestos cuando son importados de México, como bienes terminados (USMCOC, 2012). Aunado a estos regímenes fiscales preferentes, también la construcción de infraestructura, como los parques industriales y servicios públicos, entre otros, han sido factores de atracción para la IED. Este comportamiento de la inversión extranjera directa en términos territoriales muestra también cómo, desde finales de los ochenta, la atracción de la IED es un elemento clave de la estrategia de desarrollo puesta en marcha por el gobierno mexicano (Dussel-Peters, 2000).

Como resultado del análisis anterior, se advierte que en 1994 los estados de la frontera norte recibían únicamente el 20 por ciento de la IED que se derramaba a México, 
pero hacia 2000, esta participación se incrementó al 31 por ciento, lo cual es un porcentaje significativo de casi el doble de la inversión. El promedio para el periodo ha sido del 26 por ciento, a raíz de la caída de los flujos de IED en 2001. Entre los estados de la frontera más favorecidos por esta inversión se encuentran Nuevo León, Chihuahua y Baja California. Por último, si se consideran los flujos de IED aplicados a la maquiladora asentada en los estados fronterizos del norte de México, entonces los porcentajes de participación se elevan a casi el 50 por ciento para el promedio del periodo. Ello da cuenta de la importancia de la actividad maquiladora en el desempeño económico de estos estados.

De nueva cuenta, hay evidencia de la fuerte dependencia económica de México respecto de su país vecino, en términos de recepción de la IED, pues de Estados Unidos proviene la mayor parte del capital invertido y esta participación se encuentra sujeta a las condiciones económicas, así como a la relación económico-política entre ambos países.

\section{RefLeXIONES FINALES}

En los apartados anteriores se presentó un análisis sobre el contexto de la interdependencia económica entre México y Estados Unidos, con base en la evolución de las principales variables que reflejan esta dinámica. Para ello fue necesaria una previa revisión de la literatura teórico-metodológica, lo que nos ha permitido rescatar diferentes aproximaciones de la interdependencia y su medición.

Entre los principales hallazgos, ha sido posible identificar que un incremento en las relaciones comerciales entre los países trae consigo una disminución en el conflicto cuando los costos de estas relaciones son controlados, en tanto que en las relaciones donde existe interdependencia extrema es posible que las situaciones de conflicto aumenten.

Asimismo, de esta revisión destacamos los conceptos de sensibilidad y vulnerabilidad, en la medida de lo que se esperaría de la relación de interdependencia entre México y Estados Unidos. Estos conceptos nos han permitido analizar los coeficientes de interdependencia, más allá de sus valores como flujos comerciales, para de esta manera calificar el carácter de esta relación comercial. Además de corroborar la dependencia de las exportaciones mexicanas respecto del mercado estadunidense, cabe agregar que tal dependencia es vulnerable, ya que cambios positivos o negativos en dicho mercado acarrean consecuencias positivas o negativas en la demanda de productos mexicanos. Aún más, en términos de una medida de sensibilidad, México se encuentra en una posición desventajosa frente a Estados Unidos, como se ha 
reflejado en la forma en que cada país ha afrontado las crisis económicas. Por último, la dependencia o intensidad de las exportaciones revela que la interdependencia es asimétrica, ya que las exportaciones mexicanas no son significativas para el mercado de Estados Unidos.

Por otro lado, cuando se analizan las importaciones hechas por México de los bienes procedentes de Estados Unidos, se comprueba que se mantiene una concentración geográfica de las importaciones. Si bien cabría matizar que esa concentración ha disminuido en los últimos diez años, el mercado mexicano aún es significativo para los productos estadunidenses. Este hecho, aunado a la intensidad de las importaciones, da como resultado que exista una dependencia de las importaciones estadunidenses. En esa medida, se genera de nuevo una posición de vulnerabilidad para México, mas no para Estados Unidos, ya que lo que este último destina a México no constituye un porcentaje significativo del total enviado al resto del mundo.

En este artículo se ha considerado como variable complementaria para el análisis de la interdependencia económica la presencia de flujos de IED, por ser Estados Unidos la principal fuente de esos flujos. Uno de los resultados a destacar ha sido que, gracias a la flexibilización de las leyes de inversión extranjera, la entrada de capitales de Estados Unidos incrementó, de manera particular para la industria de la transformación, así como para la producción maquiladora. En el aspecto regional, los estados de la frontera norte han sido, en términos relativos, los principales receptores de este flujo, pues la producción maquiladora se concentra en esa franja. Los conceptos de vulnerabilidad y sensibilidad sí se aplican en este caso, pues se ha visto cómo los cambios en la dinámica de la economía estadunidense han impactado en una disminución de la inversión.

Por todo lo anterior, se concluye que, derivado de la relación económica, política y social entre México y Estados Unidos, se pone en evidencia la necesidad de la construcción de una agenda bilateral que atienda no sólo los problemas comunes entre ambos países, sino que además defina las directrices para una mayor cooperación económica en la región, con miras en el avance de la disminución de las asimetrías entre estos países vecinos.

En esa medida, es relevante conjuntar esfuerzos (academia y gobierno) para identificar los sectores y áreas donde es posible mejorar los términos de interdependencia que se traduzcan en una relación más simétrica entre ambos países. 


\section{BibLiografía}

Acevedo, ERnesto

2002 "Causas de la recesión en la industria maquiladora", Momento Económico, noviembre-diciembre, pp. 11-25.

ArJona, Luis y Kurt Unger

1996 "Competitividad internacional y desarrollo tecnológico: la industria manufacturera frente a la apertura comercial", Economía Mexicana, nueva época, vol. 5, no. 2, pp. 187-220.

AsPe, Pedro

1993 El camino mexicano de la transformación económica, México, FCE.

\section{AtKinson, GLen}

1998 "Regional Integration in the Emerging Global Economy: The Case of NAFTA", The Social Science Journal, vol. 35, no. 2, pp. 159-168.

Barajas, María del Rosío

2000 "The Global Productive Networks in the Electronics Industry: The Experience in the Binational Region Tijuana-San Diego", School of Social Sciences, University of California, Irvine, tesis doctoral.

BARBIERI, KATHERINE

1996 "Economic Interdependence: A Path to Peace or a Source of Interstate Conflict?", Journal of Peace Research, vol. 33, no. 1, pp. 29-49.

Cañas, Jesús, R. Coronado y R. Wilmer

2004 "Maquiladora Downturn: Structural Change or Cyclical Factors?", International Business and Economics Research Journal, vol. 3, no. 8, pp. 27-46.

CASAR, José I.

1989 Transformación en el patrón de especialización y comercio exterior del sector manufacturero mexicano, 1978-1987, México, Nacional Financiera, sNC-Instituto Latinoamericano de Estudios Transnacionales. 
Dussel-Peters, EnRiQue

2000 "La inversión extranjera en México. División de desarrollo productivo y empresarial", CEPAL, no. 80, octubre.

Fairlie, Alan y Sandra Queija de la Sotta

2007 ¿Relaciones económicas Perú-Chile: Integración o conflicto?, Lima, Centro de Investigaciones Sociológicas, Económicas, Políticas y Antropológicas.

FONSECA, FABRICIO

2010 "Reflejos de la interdependencia económica en las relaciones China-Estados Unidos durante la primera década del siglo xxI", Asociación Latinoamericana de Estudios de Asia y África, XIII Congreso Internacional de ALADAA.

GARZA, Humberto

1996 "La política exterior de México: entre la dependencia y la diversificación", Foro Internacional, vol.36, no. 4, pp. 641-666.

GASIOROWSKI, MARK

1986 "Economic Interdependence and International Conflict: Some Cross-national Evidence", International Studies Quarterly, vol. 30, no. 1, pp. 23-38.

Gruben, William

2004 "Have Mexico's Maquiladoras Bottomed Out?", Southwest Economy, enerofebrero, pp. 14-15.

\section{HANSON, GORDON}

2001 “U.S. Mexico Integration and Regional Economies. Evidence from BorderCity Pair?", Journal of Urban Economics, vol. 50, no. 2, pp. 259-287.

1996 "Economic Integration, Intra-Industry Trade and Frontier Regions", European Economic Review, vol. 40, nos. 3-5, pp. 941-949.

HANSON, GORDON y RAYMOND ROBERTSON

2008 "China and the Manufacturing Exports of Other Developing Countries", NBER (documento de trabajo, 14497).

HARRISON, R.

1988 "Economic Interdependence, Bargaining Power, and Political Influence", International Organization, vol. 42, no. 3, pp. 461-483. 
HERNÁNDEZ, RoBERTO H.

2012 "Economic Liberalization and Trade Relations between Mexico and China", Journal of Current Chinese Affairs, vol. 41, no. 1, pp. 44-96, <http://journals. sub.uni-hamburg.de/giga/jcca/issue/view/74>.

Hirschman, Albert O.

1945 National Power and the Structure of Foreign Trade, Berkeley, University of California Press.

INTERNATIONAL TRADE ADMINISTRATION

2012 "Building a North American Community", Council on Foreign Relations-Canadian Council of Chief Executives-Consejo Mexicano de Asuntos Internacionales, en <http: / / tse.export.gov>, consultada el 18 de septiembre de 2013.

Keohane, Robert O. y Joseph S. Nye

1989 Power and Interdependence, $2^{\text {a }}$ ed., Glenview, Ill., Little Brown.

Kroll, John A.

1993 "The Complexity of Interdependence", International Studies Quarterly, vol. 37, no. 3, septiembre, pp.321-347.

LUSTIG, NORA

1995 The Mexican Peso Crisis: The Foreseeable and the Surprise, Washington, D.C., The Bookings Institution Press.

Mundra, Kusum y J. Gerber

2010 "Is the Downturn in Maquiladora Employment Cyclical or Structural?", Indian Growth and Development Review, vol. 3, no. 1, pp. 21-35.

OCDE

1996 Trade Liberalization Policies in Mexico, París, OCDE.

PÉRez, CuAuHTÉmoc

2006 "Crisis y recuperación de la industria maquiladora de exportación 2000-2004", Revista de Análisis Económico, vol. 21, no. 48, pp. 229-256.

Philips, Keith y Jesús Cañas

2004 Business Cycle Coordination along the Texas-Mexico Border, Dallas, Federal Reserve Bank of Dallas. 
SeCretaría de ECONOMía (SE)

$<$ www.economia.gob.mx>.

SOTOMAYOR, MARITZA

2008 "Medición del comercio intraindustrial no maquilador de México", Investigación Económica, vol. 68, no. 268, pp. 39-68.

Turner, ERnesto y Alejandro DíAZ

2009 "Desarrollo e integración del norte de México y el sur de Estados Unidos", Análisis Económico, vol. 24, no. 57.

\section{UNITED NATIONS}

2013 Commodity Trade Statistics, en <http:/ / comtrade.un.org/ >, consultada el 18 de febrero de 2013.

United States-México Chamber of Commerce (usmcoc)

2012 "The North American Free Trade Agreement (NAFTA) at Five Years: What it Means for the U.S. and Mexico", en $<$ www.usmcoc.org $>$, consultada el 9 de octubre de 2013.

Vega, Gustavo

2010 "2010: balance y perspectivas del libre comercio entre México y Estados Unidos", Foro Internacional, vol. 50, no. 2, pp. 269-324.

Villareal, M. Ángeles

2010 NAFTA and the Mexican Economy, Washington, D.C., Congressional Research Service.

VILLARREAL, RENÉ

1997 Industrialización, deuda y desequilibrio externo en México. Un enfoque neoestructuralista (1929-1997), Economía Latinoamericana, México, FCE.

ZHANG, MiNG

1995 Major Powers at a Crossroads: Economic Interdependence and an Asia Pacific Security Community, Boulder, Colo., Lynne Rienner. 